\title{
Peripheral intravenous catheter-associated Staphylococcus aureus bacteraemia: more than 5 years of prospective data from two tertiary health services
}

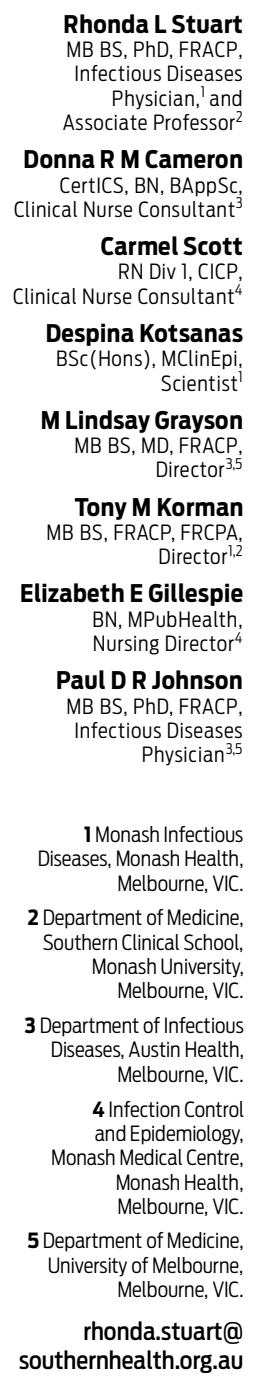

MJA 2013; 198: 551-553 doi: 10.5694/mjal2.11699

Editorial p 519 taphylococcus aureus bacteraemia (SAB) is an important hospital-acquired infection often associated with indwelling devices. ${ }^{1-3}$ The risk of a peripheral intravenous catheter (PIVC) leading to $\mathrm{SAB}$ is low, estimated to occur in about $0.1 \%$ of lines. ${ }^{4}$ However, up to $80 \%$ of hospitalised patients have a PIVC in situ at some time during their acute stay, ${ }^{5,6}$ so absolute numbers of PIVC-associated SAB may be a more pressing issue than has been previously recognised. ${ }^{1,3,5}$

Debate over the need for routine PIVC replacement is ongoing. In 1996, the United States Centers for Disease Control and Prevention recommended that PIVC sites should be rotated at intervals of 48-72 hours. ${ }^{7}$ Later guidelines suggested that routine PIVC changes were not required more frequently than 72-96 hours in adults. $^{8}$ In 1998, researchers reported that the hazard for PIVC complications (thrombophlebitis, infection) did not appear to increase during prolonged catheterisation and recommended that routine replacement was unnecessary. 9 These findings have been recently echoed in a multicentre randomised trial involving over 3200 patients but remain controversial. ${ }^{10}$

We sought to review 5 years of PIVC-associated SAB in two tertiary referral health services to define the frequency, mortality and associated risk factors for this health care-associated complication.

\section{Methods}

Two tertiary referral health services in Melbourne (Monash Health and Austin Health, with 2150 and 840 beds, respectively) were included in our analysis. Both services prospectively used the same data collection tool to collect data on every episode of $\mathrm{SAB}$ that occurred within each facility. The data for this study

\begin{abstract}
Abstrac
Objectives: To determine the incidence, risk factors for and outcomes of Staphylococcus aureus bacteraemia (SAB) associated with peripheral intravenous catheters (PIVCS).

Design, setting and patients: A review of prospectively collected data from two tertiary health services on all health care-associated SAB episodes occurring in adults aged > 17 years from January 2007 to July 2012.
\end{abstract}

Main outcome measures: Numbers of health care-associated SAB episodes; device type, location of insertion, device dwell time and outcome at 7 and 30 days for all SAB episodes associated with use of a PIVC; rates of SAB per 10000 occupied bed-days (OBDs).

Results: Overall, 137 of 583 health care-associated-SAB episodes (23.5\%) were deemed to be PIVC associated, with an incidence of 0.26/10 000 OBD. The mean dwell time for PIVCs was 3.5 days (range, $0.25-9$ days) and $45.2 \%$ of SABs occurred in PIVCs with a dwell time $\geqslant 4$ days. Of the PIVC-associated SAB episodes, 39.6\% involved PIVCs inserted in the ED, 39.6\% involved PIVCs inserted on wards and $20.8 \%$ involved PIVCs inserted by the ambulance service. Of the PIVC-associated SABs occurring within 4 days of insertion, $61 \%$ were inserted by ED staff or the ambulance service. PIVC-associated SAB were associated with a 30 -day all-cause mortality rate of $26.5 \%$.

Conclusion: PIVC-associated SAB is an under-recognised complication. The high incidences of SAB associated with PIVCs inserted in emergency locations and with prolonged dwell times support recommendations in clinical guidelines for routine removal of PIVCs.

included all health care-associated SAB episodes occurring in adults aged $>17$ years at each site from January 2007 to July 2012.

Every positive blood culture for $S$. aureus was investigated by infection control and classified as health care or community associated. Health care-associated SAB was defined as isolation of $S$. aureus from one or more blood cultures taken:

- during hospitalisation, 48 hours or more after admission; or

- within 48 hours of admission in a patient with an indwelling medical device; or

- within the last 30 days in a patient who has had surgery and the SAB is deemed to be related to the procedure; or

- within 48 hours of an invasive procedure.

Data collected for each health care-associated SAB episode included patient demographics, place of acquisition (community or hospital), likely source of infection, pri- mary clinical manifestation and outcomes at 7 and 30 days. Neither service has a dedicated peripheralline management team.

For SAB deemed to be devicerelated, details of device type, place of insertion (ambulance, emergency department [ED], ward) and device dwell time (days before the SAB was identified) were collected. A case of PIVC-associated SAB was defined as a health care-associated $\mathrm{SAB}$ in a patient:

- with a PIVC in situ or removed within the 7 days before the positive blood culture; and

- with no other source of SAB identified and either a physician or nurse documenting the PIVC as the source of the $\mathrm{SAB}$ in the medical record; and/or

- with physical findings suggesting a PIVC as the source (erythema, induration, phlebitis, tenderness).

To estimate the PIVC-associated $\mathrm{SAB}$ rate we used occupied bed-days (OBD) for all overnight stays as the denominator. 
1 Sources of health care-associated Staphylococcus aureus bacteraemia ( $\mathrm{SAB}$ ) in two tertiary health services, January 2007 to July 2012

\begin{tabular}{lccc} 
& \multicolumn{3}{c}{ SAB episodes, no. (\%) } \\
\cline { 2 - 4 } Source & $\begin{array}{c}\text { Monash } \\
\text { Health }\end{array}$ & $\begin{array}{c}\text { Austin } \\
\text { Health }\end{array}$ & Total \\
\hline Device-associated & $267(67.6 \%)$ & $107(56.9 \%)$ & $374(64.2 \%)$ \\
PIVC & $96(24.3 \%)$ & $41(21.8 \%)$ & $137(23.5 \%)$ \\
Central line & $78(19.7 \%)$ & $24(12.8 \%)$ & $102(17.5 \%)$ \\
PICC & $47(11.7 \%)$ & $12(6.4 \%)$ & $59(10.1 \%)$ \\
Haemodialysis & $27(6.8 \%)$ & $15(8.0 \%)$ & $42(7.2 \%)$ \\
Urinary device & $9(2.3 \%)$ & $3(1.6 \%)$ & $12(2 \%)$ \\
Orthopaedic device & $3(0.8 \%)$ & $6(3.2 \%)$ & $9(1.5 \%)$ \\
Other device & $7(1.8 \%)$ & $6(3.2 \%)$ & $13(2.2 \%)$ \\
Not device-associated & $128(32.4 \%)$ & $81(43.1 \%)$ & $209(35.9 \%)$ \\
Total & 395 & 188 & 583 \\
\hline
\end{tabular}

PIVC = peripheral intravenous catheter. $\mathrm{PICC}=$ peripherally inserted central catheter.

This study was approved at both hospitals by their respective ethics committees as a quality study.

\section{Results}

There were 583 health care-associated SAB episodes across the two health services, with 137 (23.5\%) deemed to be PIVC-associated SAB (Box 1). The total number of episodes of PIVCassociated $\mathrm{SAB}$ exceeded that of $\mathrm{SAB}$ associated with central intravenous lines (102 [17.5\%]).

Over the period of the study there were 5235560 total OBDs across both sites and an overall rate of PIVCassociated SAB of $0.261 / 10000$ OBD (Box 2). Of the patients with PIVCassociated $\mathrm{SAB}, 95$ (69\%) were men and mean age was 71 years (range, $18-95$ years).
Dwell time could be ascertained in 124 of the 137 episodes of PIVCassociated SAB. Of these, the mean PIVC dwell time was 3.5 days (median, 3 days; range, 0.25-9 days). Fifty-six PIVC-associated SAB episodes (45.2\%) occurred in PIVCs with dwell times greater than or equal to 4 days (Box 2).

There were 44 PIVCs (39.6\%) inserted in the ED, $44(39.6 \%)$ in the ward and $23(20.8 \%)$ by the ambulance service (Box 2). Of 68 PIVCassociated $\mathrm{SAB}$ episodes occurring within 3 days of insertion, 24 (35.3\%) were inserted in the ED or by the ambulance service. This increased to $61 \%$ (48 of $79 \mathrm{SAB}$ episodes) for dwell times of $\leqslant 4$ days.

PIVC-associated SAB episodes were associated with a 30-day allcause mortality rate of $26.5 \%$.

\section{Discussion}

Data collected over more than 5 years in two tertiary health services showed a high incidence of SAB episodes associated with PIVCs inserted in emergency locations and with prolonged ( $\geqslant 4$ days) dwell times.

$\mathrm{SAB}$ is a major cause of morbidity and mortality. Similar to our finding of $26.5 \%$, a 30 -day all-cause mortality rate of $20.6 \%$ has been reported previously in a study of 1994 episodes of SAB. ${ }^{3}$

Each PIVC-associated SAB episode also has a significant financial cost. Financial data for SAB in Australia are scarce, and measuring the economic costs were beyond the scope of this study. Assumed additional costs of
2 Details of PIVC-associated Staphylococcus aureus bacteraemia in two tertiary health services, January 2007 to July $2012 *$

\begin{tabular}{lccc} 
Variable & Monash Health & Austin Health & Total \\
\hline Rate per 10 000 OBD & 0.288 & 0.280 & 0.261 \\
MRSA episodes & $18 / 96(18.8 \%)$ & $14 / 41(34.1 \%)$ & $32 / 137(23.4 \%)$ \\
Location where insertion took place & & & \\
Ambulance & $20 / 77(26.0 \%)$ & $3 / 34(8.8 \%)$ & $23 / 111(20.8 \%)$ \\
ED & $28 / 77(36.4 \%)$ & $16 / 34(47.1 \%)$ & $44 / 111(39.6 \%)$ \\
Ward & $29 / 77(37.7 \%)$ & $15 / 34(44.1 \%)$ & $44 / 111(39.6 \%)$ \\
Mean dwell time, days (range) & $3.5(1-9)$ & $3.5(0.25-9)$ & $3.5(0.25-9)$ \\
Dwell time $\geqslant 4$ days & $43 / 90(47.8 \%)$ & $13 / 34(38.2 \%)$ & $56 / 124(45.2 \%)$ \\
Alive at 7 days & $89 / 96(92.7 \%)$ & $38 / 41(92.7 \%)$ & $127 / 137(92.7 \%)$ \\
Alive at 30 days & $48 / 61(78.7 \%)$ & $27 / 41(65.9 \%)$ & $75 / 102(73.5 \%)$ \\
\hline
\end{tabular}

OBD = occupied bed-days. MRSA = methicillin-resistant $S$. aureus. ED = emergency department. $*$ Values are no. of episodes/total PIVC-associated SAB episodes (\%) unless otherwise indicated. Denominators vary according to the total number of episodes that could be ascertained for each variable.
\$20 000 per episode have been previously quoted. ${ }^{2}$ This would equate to $\$ 29500$ on average, adjusted for the consumer price index, and give an estimated total cost of PIVC-associated $\mathrm{SAB}$ at our two institutions for the study period of $\$ 4.04$ million.

Reducing PIVC-associated SAB is therefore paramount. Prevention strategies include not inserting PIVCs unless needed, ensuring aseptic technique on insertion and early removal when the line is no longer required or when it is inserted during emergency situations. Additionally, reduction of $\mathrm{SAB}$ may also be possible by routine replacement of PIVCs. ${ }^{78}$ A multicentre, randomised, non-blinded equivalence trial among adults with a PIVC of expected use longer than 4 days was reported recently. Researchers compared rates of thrombophlebitis, during catheterisation or within 48 hours of removal, between patients who had PIVCs routinely replaced on Day 3 and those having a PIVC replaced only if clinically indicated (completion of therapy, thrombophlebitis, inflammation, occlusion, suspected infection). While the study was powered for phlebitis, they only noted one PIVC-associated SAB (in the routine-change arm) in 3283 patients and out of 5907 catheters. $^{10}$

Our study design was based on investigation of each incident case of $\mathrm{SAB}$, not following two arms of a randomised cohort. We believe our data suggest that timely removal of PIVCs is important for reducing risk of $S A B$, particularly when inserted in suboptimal circumstances. The likely explanation for the different finding of the equivalence trial is that the true incidence of PIVC-associated SAB was below the level of detection allowed by its design, and that phlebitis is not a reliable predictor of SAB.

Other factors that might have influenced the results of the equivalence study include non-blinding of the research nurses, the daily presence of research nurses on the wards and up to $40 \%$ of PIVC being placed by a specialised team dedicated to inserting intravenous catheters. ${ }^{10}$ Our study, on the other hand, shows rates of PIVC-associated SAB in a real-life situation, where PIVCs were inserted in EDs and wards. 
From our study, we cannot confirm whether PIVC replacement at 72-96 hours is appropriate or whether only clinically indicated replacement is warranted. However, about $45 \%$ of PIVC-associated SAB were potentially preventable by removal before the 4day cut-off.

Strengths of our study include the large numbers of cases across two sites and the prospective nature of data collection. Limitations include the fact that the rate of PIVC-associated SAB we have defined is a crude rate. To adequately define relative risks associated with longer dwell times we would need data on PIVC days among this population. Additionally, although to our knowledge these data are the largest series of PIVC-associated SAB episodes reported, the results may not be generalisable to all health services.
Our study highlights the significant issue of PIVC-associated SAB. Further studies powered for the outcome of $\mathrm{SAB}$ to solve the issue of whether routine PIVC replacement reduces $\mathrm{SAB}$ are required, as are studies investigating the cost of SAB. In the meantime, protocols for PIVC removal at $\leqslant 4$ days will remain at our two hospitals. National standards for PIVC insertion and maintenance are needed.

Competing interests: No relevant disclosures.

Received 20 Nov 2012, accepted 13 Mar 2013.

1 Trinh TT, Chan PA, Edwards O, et al. Peripheral venous catheter-related Staphylococcus aureus bacteremia. Infect Control Hosp Epidemiol 2011; 32: 579-583.

2 Collignon PJ, Dreimanis DE, Beckingham WD, et al. Intravascular catheter bloodstream infections: an effective and sustained hospitalwide prevention program over 8 years. Med J Aust 2007; 187: 551-554.

3 Turnidge JD, Kotsanas D, Munckhof W, et al; Australia New Zealand Cooperative on Outcomes in Staphylococcal sepsis. Staphylococcus aureus bacteraemia: a major cause of mortality in
Australia and New Zealand. Med J Aust 2009; 191: 368-373.

4 Maki DG, Kluger DM, Crnich CJ. The risk of bloodstream infection in adults with different intravascular devices: a systematic review of 200 published prospective studies. Mayo Clin Proc 2006; 81: 1159-1171.

5 Pujol M, Hornero A, Saballs M, et al. Clinical epidemiology and outcomes of peripheral venous catheter-related bloodstream infections at a university-affiliated hospital. J Hosp Infect 2007; 67: 22-29.

6 Maki DG. Improving the safety of peripheral intravenous catheters. BMJ 2008; 337: a630.

7 Pearson M. Guidelines for prevention of intravascular device-related infections. Hospital Infection Control Practices Advisory Committee. Infect Control Hosp Epidemiol 1996; 17: 438-473.

8 O'Grady NP, Alexander M, Burns LA, et al; Healthcare Infection Control Practices Advisory Committee. Guidelines for the prevention of intravascular catheter-related infections. Clin Infect Dis 2011; 52: el62-el93.

9 Bregenzer T, Conen D, Sakmann P, Widmer AF. Is routine replacement of peripheral intravenous catheters necessary? Arch Intern Med 1998; 158 : 151-156.

10 Rickard CM, Webster J, Wallis MC, et al. Routine versus clinically indicated replacement of peripheral intravenous catheters: a randomised controlled equivalence trial. Lancet 2012; 380: 1066-1074. 